\title{
Plasma internal profile control using IDA-PBC: Application to TCV
}

\author{
N.M.Trang VUª ${ }^{\star a}$ R. NOUAILLETAS ${ }^{\mathrm{a}}$, E. MALJAARS ${ }^{\mathrm{b}}$, F. FELICI $^{\mathrm{b}}$, O.SAUTER $^{\mathrm{c}}$, TCV Team $^{\mathrm{c}}$ \\ ${ }^{a}$ CEA, IRFM, F-13108 Saint-Paul-Lez-Durance, France \\ ${ }^{b}$ Eindhoven University of Technology, Faculty of Mechanical Engineering, Control Systems Technology Group, P.O. Box 513, 5600 MB \\ Eindhoven, The Netherlands \\ ${ }^{c}$ École Polytechnique Fédérale de Lausanne (EPFL), Swiss Plasma Center (SPC), CH-1015 Lausanne, Switzerland
}

\begin{abstract}
In this paper, new results of plasma $\iota$-profile and $\beta$ control on TCV, using total plasma current $I_{p}$, and ECCD (Electron Cyclotron heating and Current Drive) heating source have been discussed. The control model is governed by the resistive diffusion equation coupled with the thermal transport equation, written in PCH (Port-Controlled Hamiltonian) formulation. The IDA-PBC (Interconnection and Damping Assignment - Passivity based Control) controller is developed and tested on simulation as well as on TCV real plant. Two test scenarios are considered: $\iota$ control only, and $\iota$ and $\beta$ control. The spatial distributions of ECCD profiles are pre-defined and only input powers are used for control design. Thus, a stationary control is defined in order to consider all non-linearity and actuator constraint, and a linear feedback IDA-PBC will ensure the convergence speed and the robustness of the closed-loop system. The obtained results are encouraging towards using routinely such plasma advanced control algorithm in a near future.
\end{abstract}

Keywords: Tokamak plasma control, plasma current profile control, Port-Controlled Hamiltonian systems, IDA-PBC control

\section{Introduction}

The control of plasma current density profile or $\iota$-profile and $\beta$ (a measure of the temperature) is a key issue to achieve advanced plasma scenarios with high repeatability. The 1D resistive diffusion equation of the magnetic flux [2, Chap.6] is commonly used for the plasma current profile control, such as model predictive control [6]; optimal tracking problem [8]; passivity using Lyapunov approach [1], or sliding mode [4], etc. Among these control strategies, the IDA-PBC (Interconnection and Damping Assignment - Passivity based Control) based on PCH (PortControlled Hamiltonian) tokamak system is a promising method which has been originally developed for WEST [10]. The extended model that includes MHD couplings as well as thermal diffusion for a better plasma current profile control is also studied in [11] with the application to TCV.

The purpose of this paper is to discuss about new simulation and experimental results of the IDA-PBC controller on TCV. The developed controller allows to stabilize $\iota$ profile and $\beta$ around the desired references by three actions: plasma total current $I_{p}$, distributed non inductive current-drive $J_{\text {ext }}$ and external heating source $S_{\text {heat }}$.

Email addresses: ngoc-minh-trang.vu@cea.fr (N.M.Trang $\left.\mathrm{VU}^{\star}\right)$, remy.nouailletas@cea.fr (R. NOUAILLETAS), E.Maljaars@tue.nl (E. MALJAARS), F.Felici@tue.nl (F. FELICI), olivier.sauter@epfl.ch (O.SAUTER)
Two scenarios are considered in TCV experiments. The first one is $\iota$ control only case where only resistive diffusion equation is used with two control actions $I_{p}$ and $J_{\text {ext }}, S_{\text {heat }}$ is pre-defined by the plasma scenario. In the second one, non-linear MHD couplings and thermal-electromagnetic model are investigated, the control law thus includes the total heating power $S_{\text {heat }}$.

The main idea of IDA-PBC is recalled in the next section. In Section 3 the controlled plant is described, as well as the governing equations for the resistive diffusion and the thermal diffusion problems written in $\mathrm{PCH}$ formulation. In Section 4, the control issues of the considered system are presented, a control strategy is proposed to overcome these problems. Simulation results using RAPTOR code [3] for the TCV configuration are presented in Section 5. Based on these previous simulation tests, the IDA-PBC controller has been implemented and tested on the TCV real-time control system. Preliminary results are showed in Section 6. The paper ends with some conclusions and prospects for the future works.

\section{IDA-PBC closed-loop control for PCH systems}

The IDA-PBC control design [7] may be considered the most general method among Passivity Based Control (PBC) designs for PCH systems. A brief theorem of IDA$\mathrm{PBC}$ methodology is given hereafter. 
Theorem. If it exists a static IDA-PBC feedback $u_{I D A}(x)$ which transforms a canonical PCH system into a desired one:

$$
\begin{array}{r}
\dot{x}=[\mathcal{J}(x)-\mathcal{R}(x)] \frac{\partial \mathbb{H}}{\partial x}(x)+g(x) u(t) \\
\stackrel{\dot{x}}{u_{I D A}}=\left[\mathcal{J}_{d}(x)-\mathcal{R}_{d}(x)\right] \frac{\partial \mathbb{H}_{d}}{\partial x}(x)
\end{array}
$$

then the closed-loop system is stable (locally) at $x_{d}=$ arg $\min \mathbb{H}_{d}(x)$. The feedback control can be derived from the matching equation:

$$
\begin{aligned}
{[\mathcal{J}(x)} & -\mathcal{R}(x)] \frac{\partial \mathbb{H}}{\partial x}(x)+g(x) u_{I D A}(x) \\
= & {\left[\mathcal{J}_{d}(x)-\mathcal{R}_{d}(x)\right] \frac{\partial \mathbb{H}_{d}}{\partial x}(x) }
\end{aligned}
$$

where $x \in \mathbb{R}^{n}$ the energy state variables, $u, y \in \mathbb{R}^{m}$ the input - output variables; $\mathbb{H}$ Hamiltonian function (total energy); $\mathcal{J}=-\mathcal{J}^{T} \in \mathbb{R}^{n \times n}$ skew interconnection matrix, $g \in \mathbb{R}^{n \times m}$ control matrix, and $\mathcal{R}=\mathcal{R}^{T} \in \mathbb{R}_{+}^{n \times n}$ dissipation matrix; $\mathcal{J}_{d}=-\mathcal{J}_{d}^{T} ; \mathcal{R}_{d}=\mathcal{R}_{d}^{T}$ desired parameters, and $\mathbb{H}_{d}(x)$ desired total energy.

The feedback control law will be derived simultaneously with an appropriate choice of the tuning parameters $\mathcal{J}_{d}, \mathcal{R}_{d}$ and $\mathbb{H}_{d}$. The readers can refer to [7] or [10] for the discussion of matching equation solutions.

\section{Controlled plant description}

The simplified tokamak plasma model (figure 3.1 ) is a multi-physics system governed by Maxwell's equations in the electromagnetic domain, as well as energy balance equations in the material domain. The considered system is assumed quasi-static equilibrium [2, Chap. 6]. Therefore, only the thermodynamics associated with heat transport equation is presented in the material domain. Two strong couplings between electromagnetic and thermal domains are Lorentz force and Joule effect. The couplings in the resistivity $\eta$, in the thermal diffusion coefficient $\chi$ as well as in the bootstrap current $J_{b s}$ are all considered.

Figure 3.1 also presents different actuators in each domain for plasma heating: ohmic heating by inductive current at the plasma edge $I_{p}$, non-inductive current drive $J_{\text {ext }}$ and heat flow $S_{\text {heat }}$. The mass injection is used to control the plasma density by an independent system and is not considered.

The control objective is to reach a predefined $\iota$ profile and $\beta$ using $I_{p}, J_{\text {ext }}$ and $S_{\text {heat }}$.

\subsection{Resistive diffusion control model}

The so-called resistive diffusion equation [2, Chap.6] has been widely used for the plasma current density profile control, or $\iota$-profile control. An equivalent model in PCH
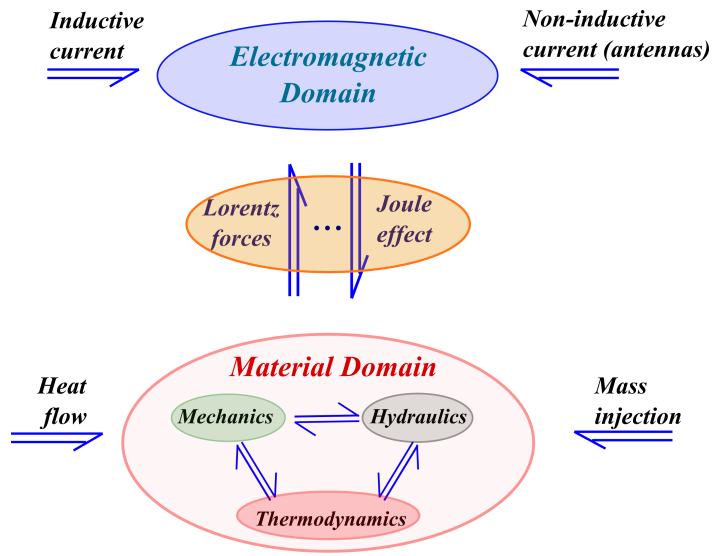

Figure 3.1: Multi-physics tokamak system

formulation was developed in our previous work[10]. Generally, this model is derived from Maxwell's equations in the normalized $1 \mathrm{D}$ coordinate $z=\rho / \rho_{\max } \in[0,1]$ :

$$
\begin{gathered}
\left(\begin{array}{c}
\partial_{t} \mathbf{d} \\
\partial_{t} \mathbf{b}
\end{array}\right)=\left[\mathcal{J}_{E M}-\mathcal{R}_{E M}(\eta)\right]\left(\begin{array}{c}
\partial_{\mathbf{d}} \mathbb{H}_{E M} \\
\partial_{\mathbf{b}} \mathbb{H}_{E M}
\end{array}\right)+\left(\begin{array}{c}
-\mathbf{J}_{e x t}-\mathbf{J}_{b s} \\
J_{4} I_{p}
\end{array}\right) \\
\mathbb{H}_{E M}=\frac{1}{2}\left(\mathbf{d}^{T} G_{e l} \mathbf{d}+\mathbf{b}^{T} G_{m g} \mathbf{b}\right)
\end{gathered}
$$

The bold variables $\mathbf{d}, \mathbf{b}, \mathbf{J}_{b s}$ and $\mathbf{J}_{\text {ext }}$ stand for the timevarying coefficients of respectively the electric field, the magnetic field, the bootstrap current density and the external current source density. The interconnection matrix $\mathcal{J}_{E M}=-\mathcal{J}_{E M}^{T}$ is obtained from the discretization (and reduction) of the spatial derivation $\partial_{z}$ in the approximation bases; $J_{4}$ relates to the boundary effect. The dissipation matrix $\mathcal{R}_{E M}$ is computed from the resistivity $\eta(z, t)$. The electromagnetic energy $\mathbb{H}_{E M}$ is defined as a quadratic function, where $G_{e l}$ and $G_{m g}$ are symmetric positive and represent, respectively, the electric permittivity $\epsilon_{0}$ and the magnetic permeability $\mu_{0}$.

\subsection{Coupled control system}

The influence of temperature $T$ on certain parameters in electromagnetic domain, such as the resistivity $\eta(T)$ and the bootstrap current $J_{b t}\left(\partial_{z} T\right)$, is not negligible. Therefore, a coupled control model including the resistive diffusion and the thermal diffusion, was investigated in [11]:

$$
\left(\begin{array}{c}
\partial_{t} \mathbf{d} \\
\partial_{t} \mathbf{b} \\
\partial_{t} \mathbf{e}_{e x} \\
0
\end{array}\right)=(\mathcal{J}-\mathcal{R})\left(\begin{array}{c}
\partial_{\mathbf{d}} \mathbb{H}_{E M} \\
\partial_{\mathbf{b}} \mathbb{H}_{E M} \\
\partial_{\mathbf{e}_{e x} \mathbb{H}_{T}} \\
\mathbf{f}_{q}
\end{array}\right)+\left(\begin{array}{c}
-\mathbf{J}_{e x t}-\mathbf{J}_{b s} \\
J_{4} I_{p} \\
\mathbf{S}_{h e a t} \\
0
\end{array}\right)
$$

where $\mathcal{J}=\left[\begin{array}{cc}\mathcal{J}_{E M} & 0 \\ 0 & \mathcal{J}_{T}\end{array}\right] ; \mathcal{R}=\left[\begin{array}{cc}\mathcal{R}_{E M}\left(\frac{1}{\eta}\right) & 0 \\ 0 & \mathcal{R}_{T}(\chi)\end{array}\right]$; $\partial_{t} \mathbf{e}_{e x}, \mathbf{f}_{q}$ and $\mathbf{S}_{\text {heat }}$ are the time-varying coefficients of respectively the entropy, the heat flux and the external heating source; $\mathcal{J}_{T}=-\mathcal{J}_{T}^{T}$ is similar to $\mathcal{J}_{E M}, \mathcal{R}_{T}=\mathcal{R}_{T}^{T} \geq 0$ is derived from the thermal diffusion coefficient $\chi$, and $\mathbb{H}_{T}$ is the thermal energy.

The MHD couplings - including the terms $J_{b s}\left(\mathbf{b}, T, \partial_{z} T\right), \quad \eta(T)$, and $\chi\left(\partial_{z} T, \mathbf{b}\right)$ are estimated 
by the empirical analytic expressions given in [9]:

$$
\left\{\begin{array}{l}
\eta(T)=C_{\eta}(\mathbf{b}) T^{-3 / 2} \\
\chi\left(\partial_{z} T, \mathbf{b}\right)=C_{\chi}(\mathbf{b}) \partial_{z} T \\
\mathbf{J}_{b s}=C_{b s}(1 / \mathbf{b}) S_{\text {heat }}
\end{array}\right.
$$

where $C_{\eta}, C_{\chi}$, and $C_{b s}$ are the coefficients depending on the system state.

Remark: Due to the different orders of magnitude between $T$ and $\mathbf{b}$ and the different timescales in plasma dynamics between heat diffusion $\left(\sim 10^{-3} s\right)$ and magnetic field radial diffusion $\left(\sim 10^{-1} s\right)$, the temperature can be considered as a static steady-state for the coupling calculation.

\section{Control issues and strategy}

Challenges in tokamak control arise not only from the PDE resolution and the non-linear MHD couplings, usually badly estimated, but also from the technological constraints of the actuators. In the considered facility, two ECCD clusters (co-current and counter-current) generate both non-inductive current $J_{\text {ext }}$ and external heating source $S_{\text {heat }}$. In practice, these distributed control signals have pre-defined deposition profiles $\left(f_{A}, f_{B}\right.$ for current drive and $f_{\text {heat }}$ for heating source). The controllable inputs are indeed the two input powers $P_{A}$ and $P_{B}$. We assume in this work that the two clusters deposit heat in the same location, which allows to approximate $S_{\text {heat }}$ by the sum of $P_{A}$ and $P_{B}$ :

$$
\left\{\begin{array}{l}
J_{\text {ext }}=f_{A}(z, t) P_{A}+f_{B}(z, t) P_{B} \\
S_{\text {heat }}=f_{\text {heat }}(z, t) P_{\text {heat }} \approx f_{\text {heat }}(z, t)\left(P_{A}+P_{B}\right)
\end{array}\right.
$$

The proposed controller in figure 4.1 first derives a stationary feedforward control from the given references, by non-linearly inverting the plasma PCH models in (3.1) or (3.2). The stationary control allows to take into account both system non-linearity and control deposition profile constraint. A linear IDA-PBC is used to improve the convergence speed of the closed-loop system. Computation details are referred to [10] and [11].

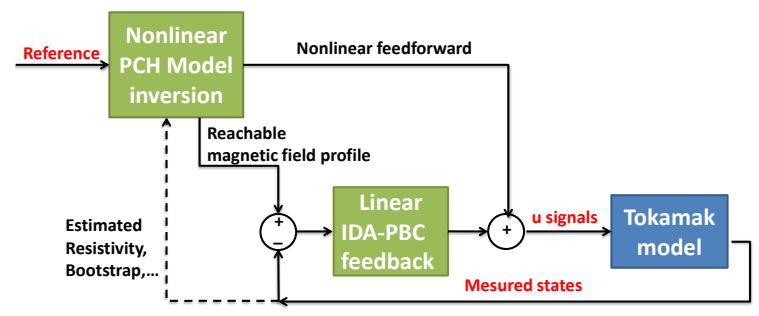

Figure 4.1: The proposed control strategy offline code [3] developed for TCV. Two test cases are considered:

- $\iota$-profile control only case: only the resistive diffusion equation (3.1) is considered, thus two actuators $I_{p}$ and $P_{\text {ext }}$ are included in the control law; $S_{\text {heat }}$ is given. In this case, we aim at two positions of $\iota$-profile $z=$ $[0.1,0.5]$.

- $\iota$-profile and $\beta$ control case: the coupled control model (3.2) is considered. All three actuators $\left(I_{p}, P_{\text {ext }}, P_{\text {heat }}\right)$ are used in order to handle two $\iota$ values (at $z=[0.1,0.5])$ and $\beta$.

Figure 5.1 shows very good simulation results for both test cases. It is obvious that the controller doesn't respect the $\beta$ reference in the first case (figure 5.1.a) since the thermal model isn't included. Otherwise, the controller can handle both $\iota$ and $\beta$ in the second test case (figure 5.1.b).
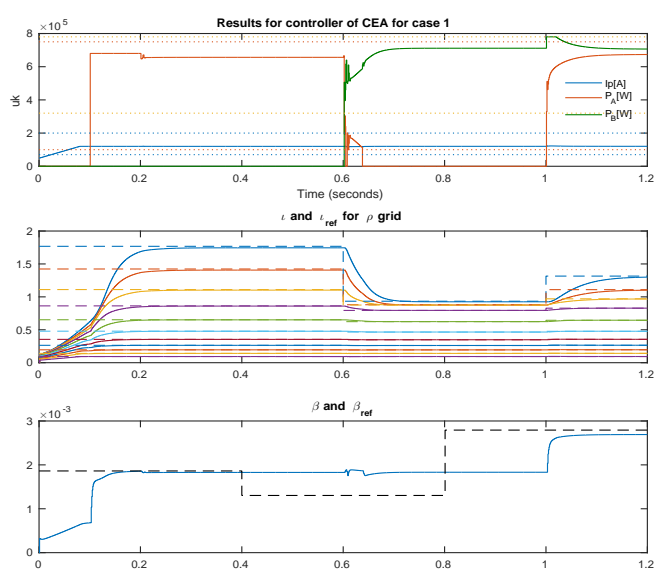

a.

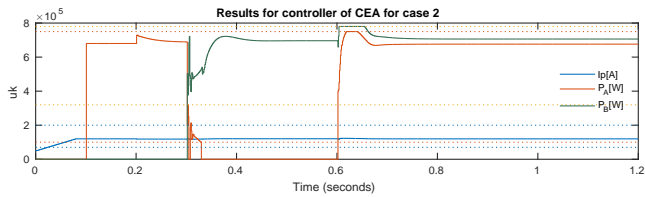

$\rightarrow$ and ${ }^{{ }_{\text {ret }}}$ for $\rho$ grid
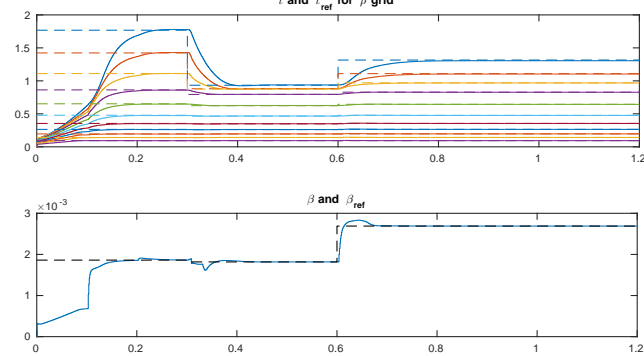

b.

Figure 5.1: IDA-PBC control for case 1 (a) and case 2 (b)

\section{Real-time experimental result}

The developed IDA-PBC controller was tested in the EUROfusion MST-1 campaign. RAPTOR real-time code
In the sequel, the $\iota$-profile and $\beta$ controls using $\mathrm{PCH}$ model and IDA-PBC method are tested with RAPTOR 
is used as observer in the TCV real-time control system (figure 6.1).

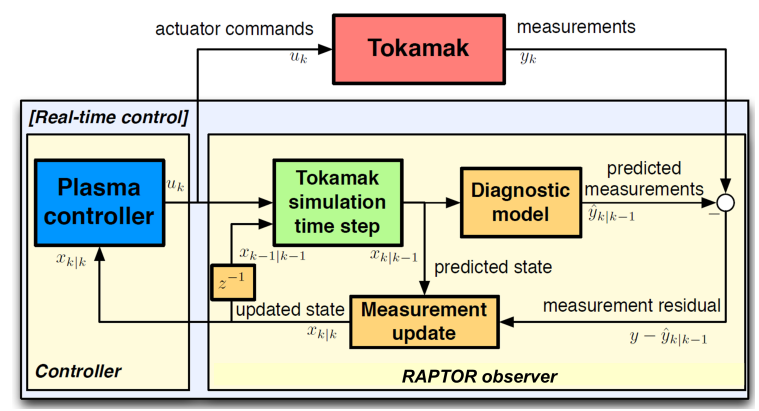

Figure 6.1: RAPTOR observer and real-time controller in Tokamak system (cf.[5])

In this occasion, we could only have one shot for the first test case: $\iota$ control only (figure 6.2). The controller was switched on on $t=[0.7 s, 1.5 s]$. Unfortunately, the $I_{p}$ control was not available for this shot. We could only regulate $P_{A}$ and $P_{B}$, hence we aimed to control the $\iota$ profile only at the position $z=0.1$. Notice that $P_{A}+$ $P_{B}$ was given in this test case. The experiment showed a pretty good result at the considered $\iota$ value. An integrator [11] was also used in order to approach the other $\iota$ values to the references.

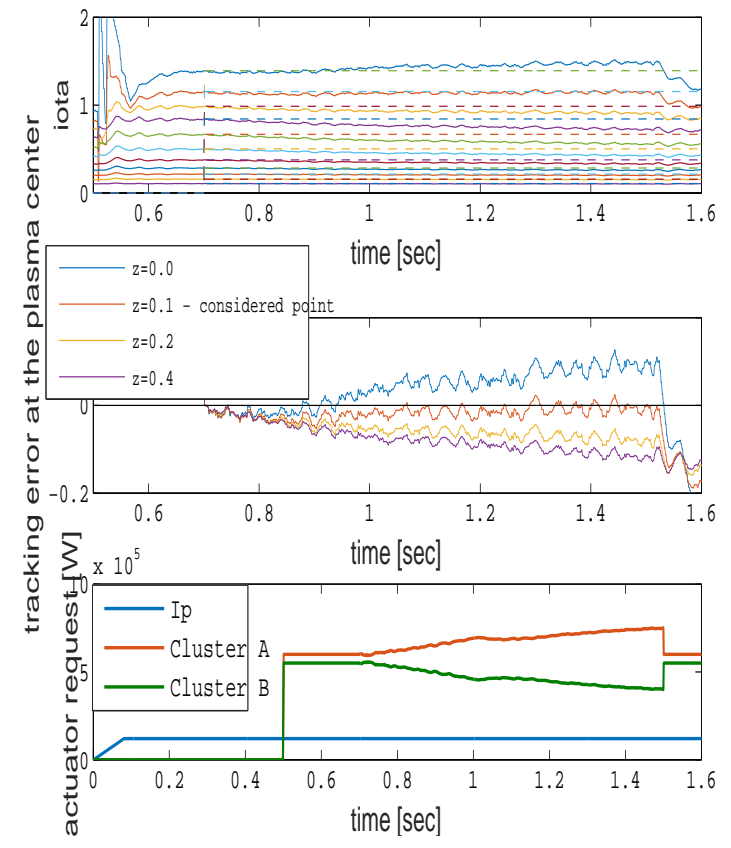

Figure 6.2: Experimental data and controller behavior (on $t=$ $[0.7 s, 1.5 s])$ of shot 53108 for $\iota$ control

\section{Conclusion}

The paper presents new results of IDA -PBC design for $\iota$-profile and $\beta$ control, applied to TCV. A control strategy is defined, which combines a non-linear stationary feedforward term, based on PCH tokamak model, in order to overcome the system non-linearity and the actuator deposition profile constraint; and a linear IDA-PBC feedback term to improve the convergence speed and the robustness. The controller has been successfully tested on RAPTOR simulation and it got a very first result in TCV experiments. Nevertheless, the control law needs to be improved and tested more on real-time tokamak systems for a routine use in the future.

\section{Acknowledgment}

This work has been carried out within the framework of the EUROfusion Consortium. The authors would like to thank S. CODA, C.GALPERTI and TCV team of SPCEPFL for their support to RAPTOR simulations and TCV experiments. The views and opinions expressed herein do not necessarily reflect those of the European Commission.

\section{References}

[1] F.B. Argomedo, C. Prieur, E. Witrant, and S. Brémond. A strict control lyapunov function for a diffusion equation with time-varying distributed coefficients. IEEE Transactions on Automatic Control (2012), 2012.

[2] J. Blum. Numerical Simulation and Optimal Control in Plasma Physics. Gauthier-Villars, New York, 1989.

[3] F. Felici, O. Sauter, S. Coda, B.P. Duval, T.P. Goodman, J.M. Moret, J.I. Paley, and the TCV Team. Real-time physics-modelbased simulation of the current density profile in tokamak plasmas. Nuclear Fusion, 51, 2011.

[4] O. Gaye, E. Moulay, S. Brémond, L. Autrique, R. Nouailletas, and Y. Orlov. Sliding mode stabilization of the current profile in tokamak plasmas. Conference on Decision and Control (2011), Orlando, Florida, USA, December 2011.

[5] D. Humphreys and et al. Novel aspects of plasma control in iter. Physics of Plasmas (1994-present), 22, 2015.

[6] E. Maljaars, F. Felici, M.R. Baar, J. Dongen, G.M.D. Hogeweij, P.J.M. Geelen, and M. Steinbuch. Control of the tokamak safety factor profile with time-varying constraints using mpc. Nuclear Fusion, 55(2)(023001), 2015.

[7] R. Ortega, A.J. van der Schaft, B. Maschke, and G. Escobar. Interconnection and damping assignment: passivity-based control of port-controlled Hamiltonian systems. Automatica, 38(4):585-596, 2002.

[8] Y. Ou, C. Xu, E. Schuster, T.C. Luce, J.R. Ferron, M.L. Walker, and D.A. Humphreys. Optimal tracking control of current profile in tokamaks. IEEE Transaction on Control Systems Technology, 19(2):432-441, March 2011.

[9] O. Sauter, C. Angioni, and Y.R. Lin-Liu. Neoclassical conductivity and bootstrap current formulas for general axisymmetric equilibria and arbitrary collisionality regime. Physic of Plasma, 6(7):2835-2839, July 1999.

[10] Trang.N.M. Vu, L. Lefèvre, R. Nouailletas, and S. Brémond. An ida-pbc approach for the control of 1d plasma profile in tokamaks. 52nd IEEE Conference on Decision and Control (2013), Florence, Italy, pages 4176 - 4181, December 10-13 2013.

[11] Trang.N.M. Vu, R. Nouailletas, L. Lefèvre, S. Brémond, and F. Felici. Ida-pbc control for the coupled plasma poloidal magnetic flux and heat radial diffusion equations in tokamaks. 19th World Congress of the International Federation of Automatic Control, Cape Town, South Africa, 19:11398-11403, August 24292014 . 